BBA 23603

\title{
Sources of urea in Neurospora
}

This paper analyzes urea accumulation in standing, minimally grown cultures of a Neurospora crassa mutant lacking urease ${ }^{\mathbf{1}}$. Urea can be produced in the arginase reaction, in which arginine is hydrolyzed to ornithine and urea ${ }^{2}$. The only other major source of urea in fungi is the allantoin pathway of purine degradation ${ }^{3,1}$. It was of interest to know the relative contributions of arginine and purine degradation to urea accumulation. Below, it is shown that a strain lacking both arginase ${ }^{5}$ and urease accumulates almost as much urea as a strain lacking only urease. Since other routes of arginine catabolism are lacking in arginase-deficient strain $\mathbf{s}^{\mathbf{5}, \mathbf{6}}$, endogenous arginine is evidently a minor source of urea. The data suggest that substantial purine turnover takes place during growth of Neurospora standing cultures.

Neurospora strains used were wild type $74 \mathrm{~A}$; strains carrying mutations ure- $I$ (ureaseless, KøLmaRK's ${ }^{1}$ allele 9) or aga (arginaseless, allele UM-go6) ${ }^{5}$; and the double mutant, ure-r, aga.

Cultures were grown in VOGEL's ${ }^{7}$ medium $\mathrm{N}$ or in $\mathrm{I} 0 \mathrm{ml}$ of a "nitrate minimal" salt mixture ${ }^{8}$ which lacked nitrate but to which alternate nitrogen sources were added. Urea was isolated from media on Dowex-50W resin columns $\left(\mathrm{H}^{+}, 0.7 \mathrm{~cm} \times \mathrm{I}_{5} \mathrm{~cm}\right)$ by the method of WATTS et al. ${ }^{0}$ and measured by the method of KORITZ AND COHEN ${ }^{10}$. Arginase was assayed according to DAVIS AND MORA ${ }^{11}$.

Growth and urea accumulation of three strains is shown in Fig. I. Urea begins to accumulate at the onset of rapid growth of mutants and continues during the stationary phase. The ure-I, aga double mutant accumulates almost as much urea as the ure-I strain. No urea can be detected in wild-type filtrates, indicating that urease normally fulfils its role completely.

Urea was measured in 96-h $u r e-I$ and $u r e-I$, aga cultures to which arginine or adenine was added at $46 \mathrm{~h}$ (Table I). The arginase levels of parallel cultures were those expected of the two genotypes. Arginine enhanced urea accumulation in ure-I by about I $8 \mu$ moles. This represents most of the $25 \mu$ moles of arginine added. No enhancement of urea accumulation was seen after arginine was added to the arginaseless culture. Adenine stimulates urea production in both strains equally, suggesting that purine catabolism yields urea.

\section{TABLE I}

UREA ACCUMULATION BY wre- $I$ AND we-I, aga AFTER ADENINE AND ARGININE ADDITIONS Cultures were grown in $25 \mathrm{ml}$ VoGEL's ${ }^{7}$ minimal medium with additions as indicated. Cultures were incubated at $32^{\circ}$. Additions $\left(25 \mu\right.$ moles) were made at $4^{6} \mathrm{~h}$ and urea and arginase activity were measured $50 \mathrm{~h}$ later.

\begin{tabular}{llcl} 
Strain & Addition & $\begin{array}{l}\text { Urea accumulated } \\
\text { (fmoles/flask) }\end{array}$ & $\begin{array}{c}\text { Arginase } \\
\text { (units/mg) }\end{array}$ \\
\cline { 2 - 3 } & None & 3.0 & - \\
& Adenine & 8.4 & 50 \\
Arge-I, aga & Arginine & 21.0 & - \\
& None & 1.9 & - \\
& Adenine & 7.5 & $<0.5$ \\
\hline
\end{tabular}

Biochim. Biophys. Acta, 2 I 5 (1970) $4 \mathrm{I}_{2-4 \mathrm{I}} 4$ 
The ability of arginine and hypoxanthine to serve as nitrogen sources for three strains was tested. The aga mutant fails to use arginine as a nitrogen source but uses hypoxanthine well. The ure-I mutant uses arginine to some extent because the ornithine liberated in the arginase reaction can be used as a nitrogen source ${ }^{11}$. However, ure- $I$ completely fails to grow on hypoxanthine as a nitrogen source. All nitrogen in hypoxanthine, which is in the purine rings, is therefore normally incorporated by a pathway of which urea is an intermediate. This is consistent with our knowledge of the enzymes of purine breakdown in Neurospora ${ }^{3,12}$ and the related fungus $A$ spergillus nidulans ${ }^{4}$. Therefore, the results suggest strongly that purine catabolism is the major source of urea in minimal cultures (Fig. I).

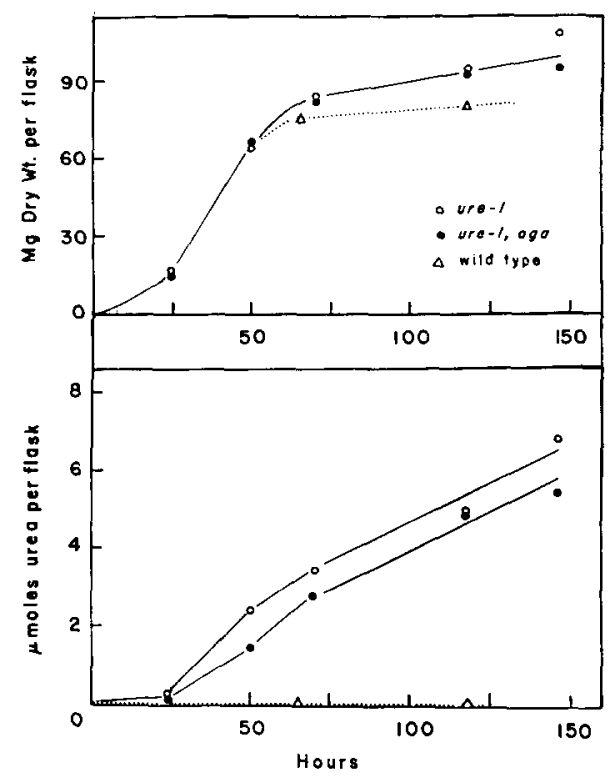

Fig. 1. Urea accumulation by ure-I; uve-I, aga (double mutant); and wild type. Cultures were grown in $25 \mathrm{ml}$ VoGEL's ${ }^{7}$ minimal medium at $32^{\circ}$ after inoculation with a drop of fresh conidial suspension. Top: increase of dry weight with time. Bottom: accumulation of urea with time. Legend is the same for both parts of the figure.

In other experiments, to be reported elsewhere, exponential cultures of the above strains were studied. In contrast to standing cultures, exponential minimal cultures accumulated little or no urea. The factors governing catabolic pathways yielding urea remain to be studied.

It is clear that urease is an important enzyme in the nitrogen economy of Neurospora. This is true even in conditions where exogenous catabolic substrates are absent and where growth is fast. The role of urease is not specific to one path, moreover, and its regulatory behavior, if any, should reflect this.

This work was supported by National Science Foundation research grant GB-64I4. I thank Loretta Port for technical assistance.

Department of Botany, University of Michigan,

Rowland H. Davis Ann Arbor, Mich. $48 \mathrm{ro4}$ (U.S.A.) 
I H. G. Kølmark, Mutation Res., 8 (I969) 5 I.

2 A. M. SRb AND N. H. Horowitz, J. Biol. Chem., I54 (1944) 229.

3 F. T. WOLF, Mycologia, 45 (1953) 825.

4 C. Scazzochio and A. J. Darlington, Biochim. Biophys. Acta, r66 (I968) 557.

5 R. H. Davis, M. B. Lawless and L. Port, J. Bacteriol., 102 (I970) 22.

6 M. Castañeda, J. Martuscelli and J. Mora, Biochim. Biophys. Acta, I4I (1967) 276.

7 H. J. VoGel, Am. Naturalist, 98 (1964) 435.

8 N. H. Horowitz, J. Biol. Chem., I 7 I (1947) 255.

9 D. C. Watts, R. L. Watts, M. D. Alexander and E. Baldwin, Biochem. J., 96 (I965) 6C.

Io S. B. Koritz and P. P. Cohen, J. Biol. Chem., 209 (I954) 145.

I I R. H. Davis And J. Mora, J. Bacteriol., 96 (I968) 383 .

i 2 R. C. Greene and H. K. Mitchell, Arch. Biochem. Biophys., 7o (1957) 603.

Received April 7th, I970

Biochim. Biophys. Acta, 2 I 5 (1970) 4I2-4I4 